\title{
GIENTests \\ Biotinidase deficiency: "if you have to have an inherited metabolic disease, this is the one to have"
}

\author{
Barry Wolf, MD, $\mathrm{PhD}^{1,2}$
}

Biotinidase recycles the vitamin biotin. Biotinidase deficiency is an autosomal recessively inherited neurocutaneous disorder. The symptoms of the disorder can be successfully treated or prevented by administering pharmacological doses of biotin. The biotinidase gene $(B T D)$ has been cloned and sequenced; its genomic organization has been determined and more than 150 mutations have been identified. The disorder meets the major criteria for newborn screening and is being universally adopted in the United States and in many countries around the world. Newborn screening will limit our understanding about the natural history of the disorder. Regardless, the disorder is an ideal example of an inherited metabolic disorder that if untreated can result in major disabilities, but if identified early can be readily treated by the oral administration of a vitamin.

Genet Med 2012:14(6):565-575

Key Words: biotin; biotinidase; biotinidase deficiency; carboxylase deficiency; mutation analysis; newborn screening

\section{INTRODUCTION}

Biotinidase (E.C. 3.5.1.12) is the enzyme that recycles the vitamin biotin. ${ }^{1,2}$ As a vitamin, biotin comes from the diet either in the free, non-protein-bound form or as small biotinylated peptides bound to protein. ${ }^{3}$ Biotin in the free form can directly enter the biotin pool and be used by the four carboxylases that use it to convert them from the inactive to the active forms. ${ }^{4}$ The carboxylases propionyl-CoA carboxylase and $\beta$-methylcrotonylCoA carboxylase are important for protein catabolism, pyruvate carboxylase is essential for gluconeogeneis, and acetyl CoA carboxylase is the first step in fatty acid synthesis. ${ }^{5}$ Protein-bound biotin must be proteolytically degraded to release biocytin (biotinyl-e-lysine) and/or small biotinyl-peptides that can be further cleaved by biotinidase, releasing the free biotin to enter the pool. ${ }^{3} \mathrm{~A}$ major function of biotinidase is to recycle biotin liberated from biocytin and/or small biotinyl-peptides from degraded holocarboxylases.

Biotinidase deficiency (OMIM\# 253260 and 609019), the major cause of late-onset biotin-responsive multiple carboxylase deficiency, is an autosomal recessively inherited neurocutaneous disorder. ${ }^{2,6,7}$ The symptoms of the disorder can be successfully treated or prevented by administering pharmacological doses of biotin. Newborn screening of the disorder is performed in most states and many countries. Frequently asked questions by parents and health-care providers and various issues about biotinidase deficiency have been addressed in a recent paper. ${ }^{8}$

\section{THE ENZYME}

Biotinidase has been shown to have biotinyl-hydrolase and biotinyl-transferase activities. ${ }^{9}$ Human biotinidase has been purified to homogeneity from plasma., ${ }^{3,10,11}$ Normal serum or plasma biotinidase is a monomeric sialylated glycoprotein with a molecular mass of $76-77 \mathrm{kDa}$. The enzyme has at least nine isoforms (four major and five minor) between $\mathrm{pH}$ 4.15 and 4.35 as observed by isoelectric focusing. ${ }^{12}$ It has six putative $\mathrm{N}$-linked glycosylation sites (arginine-X-threonine/ serine). Glycosylation of the protein could increase its mass by 13 to $19 \mathrm{kDa}$; the molecular mass of the glycosylated enzyme is estimated to be between 70 and $76 \mathrm{kDa}$, which is consistent with that of the reported glycosylated serum enzyme. ${ }^{3,10,11,13}$ Most of the microheterogeneity observed on isoelectric focusing results from differences in the degree of sialylation.

Biotinidase is a thiol enzyme that migrates to the $\alpha_{1}$-region on agarose electrophoresis. The serum enzyme has a $\mathrm{pH}$ optimum of 5-6 when biocytin or biotinyl-p-aminobenzoate (artificial substrate) is the substrate. ${ }^{1,3,11}$ Biocytin is cleaved more readily than larger biotinyl-peptides. ${ }^{3}$ Biotinidase apparently does not cleave biotin from intact holocarboxylases. The biotinyl-binding site of biotinidase is specific for the ureido group of the biotinyl moiety of various substrates. ${ }^{11,14}$ Biotinidase plays a role in the processing of dietary protein-bound biotin ${ }^{7,15}$ and has been shown to transfer biotin from biocytin to nucleophiles, such as histones ${ }^{16}$; the physiologic significance of the latter activity is not known.

The three-dimensional structure of the majority of the enzyme has been predicted through its homology with nitrilases and amidases from microorganisms. ${ }^{17}$ The active site of the enzyme involved in the cleavage of the amide bond of biocytin is similar in all the enzymes. The portion of the enzyme that is not

\footnotetext{
${ }^{1}$ Department of Medical Genetics, Henry Ford Hospital, Detroit, Michigan, USA; ${ }^{2}$ Center for Molecular Medicine and Genetics, Wayne State University, Detroit, Michigan, USA. Correspondence: Barry Wolf (bwolf1@hfhs.org)

Submitted 1 August 2011; accepted 6 September 2011; advance online publication 5 January 2012. doi:10.1038/gim.2011.6
} 
predicted by the modeling is likely important for binding the biotinyl moiety of biocytin.

\section{Biotinyl-hydrolase activity}

Polyclonal and monoclonal antibodies prepared against purified human biotinidase react on immunoblot with biotinidase in extracts of normal fibroblasts and lymphoblasts. ${ }^{9}$ These antibodies react with normal serum biotinidase that has been desialylated by treatment with neuraminidase. Individuals with profound biotinidase deficiency can be classified into at least nine distinct biochemical phenotypes on the basis of the presence or absence of cross-reacting material (CRM) to biotinidase, the number of isoforms, and the distribution frequency of the isoforms. ${ }^{18}$ The isoform patterns observed in the individuals with partial biotinidase deficiency were not different from those of individuals with profound biotinidase deficiency who had CRM.

\section{Biotinyl-transferase activity}

More than 100 children with profound biotinidase deficiency have been assessed for biotinyl-transferase activity and the presence of CRM to antibodies prepared against purified serum biotinidase ${ }^{16}$ Sera from all the symptomatic individuals studiedboth CRM-negative and CRM-positive-had no biotinyltransferase activity. Sera from children detected by newborn screening who were CRM-negative had no biotinyl-transferase activity, whereas a considerable number of the CRM-positive children had varying degrees of transferase activity. There was a statistically significant difference in biotinyl-transferase activity between the population of symptomatic enzyme-deficient children and those children who were identified by newborn screening. There was also a difference in the biotinyl-hydrolase activity between the symptomatic and newborn screening group. ${ }^{19}$

\section{MOLECULAR ORGANIZATION AND CHARACTERIZATION OF THE BIOTINIDASE GENE}

The gene for human biotinidase (BTD) is located on chromosome $3 \mathrm{q} 25 .^{20}$ The complementary DNA (cDNA) for human biotinidase from a human cDNA hepatic library has two putative ATG initiation codons and an open reading frame of $1,629 \mathrm{bp}$, relative to the first ATG codon. ${ }^{21}$ The cDNA encodes for a mature protein of 543 amino acids with a molecular mass of 56,771 daltons. The amino terminus of the mature serum biotinidase is in the same reading frame with both of the ATG codons, consistent with the two putative signal peptides.

Northern blot analysis, using a 2,000-bp probe consisting of the cDNA sequence, revealed that the biotinidase message is present in human lung, liver, skeletal muscle, kidney, pancreas, heart, brain, and placenta. ${ }^{21}$

The human gene encoding biotinidase consists of four exons, designated 1-4, with sizes of $79 \mathrm{bp}, 265 \mathrm{bp}, 150 \mathrm{bp}$, and $1,502 \mathrm{bp}$, respectively. ${ }^{22}$ Intron 1, separating exons 1 and 2, is at least $12.5 \mathrm{~kb}$, intron 2 is $6.2 \mathrm{~kb}$, and intron 3 is $0.7 \mathrm{~kb}$. Two putative translation initiation codons exist in the gene; the first is encoded within exon 1 and the other within exon 2, which contains the N-terminal methionine of the mature enzyme. The presence of an intron between the two possible initiation codons could allow for alternative splicing, which could produce transcripts encoding a protein with a 41- or a 21-residue signal peptide. ${ }^{23}$

The nucleotide sequence upstream of exons 1 and 2 has been examined for putative promoter elements. Promoter features identified from -600 to +400 are consistent with the ubiquitous expression of biotinidase with characteristics of a $\mathrm{CpG}$ island, lack of a TATA element, six consensus methylation sites, and three initiator (Inr) sequences, which are thought to be important in transcription initiation of TATA-less promoters. A consensus sequence for the liver-specific transcription factor HNF- 5 is present at -352 . The nucleotide sequence $5^{\prime}$ of exon 2 , which contains the second putative ATG initiation codon, has features associated with housekeeping genes but does contain a consensus sequence for the liver-specific transcription factor C/EBP within 300 bp of the $5^{\prime}$ end of exon 2.

\section{CLINICAL FEATURES OF BIOTINIDASE DEFICIENCY}

Clinically, children with profound biotinidase deficiency, if untreated, usually exhibit one or more of the following symptoms: hypotonia; seizures; eczematous skin rash; alopecia; respiratory problems, such as hyperventilation, laryngeal stridor, and apnea; conjunctivitis, candidiasis; ataxia; developmental delay; hearing loss; and vision problems, such as optic atrophy.,24 Metabolically, most untreated individuals with biotinidase deficiency will have one or more of the following: ketolactic acidosis, organic aciduria, and mild hyperammonemia. ${ }^{24,25}$ However, the absence of organic aciduria or metabolic ketoacidosis does not exclude the diagnosis of biotinidase deficiency in a symptomatic child.

Symptoms of untreated profound biotinidase deficiency usually appear between the ages of 1 week and 10 years, with a mean age of 3.5 months. ${ }^{25}$ Some children with biotinidase deficiency exhibit only a single symptom, whereas others have multiple neurological, cutaneous, or biochemical findings.

The most common neurologic features of individuals with untreated, profound biotinidase deficiency are seizures and hypotonia. ${ }^{2,6,25-27}$ The seizures are usually myoclonic, but may be grand mal and focal; some children have infantile spasms. ${ }^{28}$ Some untreated children have exhibited spinal cord disease characterized by progressive spastic paresis and myelopathy. ${ }^{29}$ Older affected children may develop ataxia and developmental delay. Many symptomatic children with biotinidase deficiency exhibit a variety of central nervous system abnormalities on magnetic resonance imaging or computerized tomography of the brain. ${ }^{26,28,30-32}$ These findings often improve or become normal after biotin treatment.

Sensorineural hearing loss and eye problems, such as optic atrophy, have also been described in untreated children..$^{30,33-35}$ Approximately three-quarters of untreated symptomatic children with profound biotinidase deficiency have sensorineural hearing loss that usually does not resolve or improve but remains 
static with biotin treatment. ${ }^{36}$ Many children with hearing loss can be successfully treated with hearing aids, whereas others have required cochlear implants.

Cutaneous symptoms include skin rash, alopecia, and recurrent viral or fungal infections caused by immunologic dysfunction. Respiratory problems, such as hyperventilation, laryngeal stridor, and apnea, can occur. One death initially thought to be caused by sudden infant death syndrome was subsequently attributed to biotinidase deficiency. ${ }^{37}$

Some children with profound biotinidase deficiency were asymptomatic until adolescence, when they developed sudden loss of vision with progressive optic neuropathy, scotomata, and spastic paraparesis. ${ }^{31,38,39}$ After several months of biotin therapy, the eye findings resolved and the spastic paraparesis improved. In other individuals with enzyme deficiency, paresis and eye problems have occurred during early adolescence. ${ }^{40,41}$

Children with untreated partial biotinidase deficiency may exhibit any of the above symptoms, but usually the symptoms are mild and occur only when the child is stressed, such as with a prolonged infection or by not eating. ${ }^{42,43} \mathrm{~A}$ child with partial biotinidase deficiency who was not treated with biotin exhibited hypotonia, skin rash, and hair loss during an episode of gastroenteritis at the age of $\sim 6$ months. When treated with biotin, the symptoms resolved. Individuals with partial biotinidase deficiency may develop symptoms only when exposed to a stress situation.

Almost all children with profound biotinidase deficiency become symptomatic or are at risk of becoming symptomatic, if not treated. Several adults with profound biotinidase deficiency identified through family studies have never exhibited symptoms. ${ }^{44,45}$ In addition, several enzyme-deficient siblings of symptomatic children have apparently never exhibited symptoms. It is possible that these individuals would become symptomatic if stressed.

Individuals with biotinidase deficiency who are diagnosed before they have developed symptoms (e.g., by newborn screening or because a previous child in the family was diagnosed with the disorder) and who are treated with biotin appear to have normal development. Neurological problems usually occur only in those individuals with biotinidase deficiency who have had recurrent symptoms and metabolic compromise prior to biotin treatment.

\section{TREATMENT AND MANAGEMENT}

Biotinidase deficiency is treated by supplementation with oral biotin in the free form as opposed to the protein-bound form. ${ }^{8}$ Essentially all biotin supplied as a nutrient in pharmacies and in health food stores is in the free form. Biotin therapy is lifelong. Children with biotinidase deficiency identified by newborn screening will remain asymptomatic with compliance to biotin therapy. All symptomatic children with biotinidase deficiency have improved after treatment with 5-10 $\mathrm{mg}$ of oral biotin per day; however, some of the features, such as developmental delay, optic atrophy, and hearing loss, are usually irreversible once they occur. Compliance with biotin therapy improves symptoms in symptomatic individuals. All individuals with profound biotinidase deficiency, even those who have some residual biotinidase enzyme activity, should be treated with biotin independent of their genotype ${ }^{46}$

The biochemical abnormalities and seizures rapidly resolve after biotin treatment, followed by improvement of the cutaneous abnormalities. Hair growth returns over a period of weeks to months in children who have alopecia. Optic atrophy and hearing loss may be irreversible with therapy, especially if a long period has elapsed between their onset and the initiation of treatment. Some treated children have rapidly achieved developmental milestones, whereas others have continued to have delays. Optic atrophy should be addressed with periodic ophthalmological evaluations and interventions, the hearing loss with regular audiological evaluations and hearing aids or cochlear implants, if necessary, and the developmental delays with appropriate supportive interventions and school accommodations.

There are only anecdotal reports regarding symptoms in children with partial biotinidase deficiency who were not treated with biotin. ${ }^{42}$ There have been no short- or long-term studies of those with partial deficiency. Because there is no known toxicity for biotin, children with partial deficiency are usually treated with 1-5 mg of oral biotin per day.

More data are required to determine the dosage of biotin that is necessary for older children with both profound and partial biotinidase deficiency, but essentially all children have tolerated $10 \mathrm{mg}$ of oral biotin per day with no side effects. In actuality, by keeping the dose of biotin constant as a child gets older, we are titrating these individuals; as they age, their dose of biotin per kilogram of weight is decreasing. For the most part, $10 \mathrm{mg}$ of biotin daily seems to be sufficient and the vast majority of these individuals remain asymptomatic. However, anecdotally, in my clinical experience, several girls with profound biotinidase deficiency developed hair loss during adolescence or puberty that resolved after increasing their biotin dosages from $10 \mathrm{mg}$ per day to 15 or $20 \mathrm{mg}$ per day. Perhaps both males and females require an increase in biotin dosage during and after puberty. In fact, we now routinely recommend increasing the dose in these individuals.

It is not necessary to treat individuals with protein-restricted diets. Raw eggs should be avoided because they contain avidin, an egg-white protein that binds biotin, thereby decreasing the vitamin's bioavailability. Thoroughly cooked eggs are not a problem because sufficient heating inactivates avidin, making it incapable of binding biotin.

Prior to treatment, plasma biotin concentrations of individuals with profound biotinidase deficiency may be decreased or in the low normal range. However, some methods for determining biotin concentrations measure both biotin and biotinyl conjugates, such as biocytin. Determination of biotin concentrations is of limited use in individuals with biotinidase deficiency because if a child is appropriately treated, his or her plasma and urine concentrations of biotin are always markedly elevated. Therefore, measuring biotin concentrations in 
plasma or urine is useful only in suspected cases of noncompliance where the concentrations are normal or low.

Outcome studies of children with biotinidase deficiency indicate that biotin treatment is effective in preventing symptoms. ${ }^{35,47}$ It has been stated that children with profound deficiency who have less than $1 \%$ biotinidase activity should be treated with biotin, but those with $>1 \%-10 \%$ biotinidase activity may not need treatment. ${ }^{48}$ However, a child with $1 \%-10 \%$ biotinidase activity is just as likely to develop symptoms as one with total loss of enzyme activity. ${ }^{49}$ It is, therefore, strongly recommended that all children with profound biotinidase deficiency, regardless of the residual biotinidase enzyme activity, be treated with biotin. Because genotypephenotype correlations in biotinidase deficiency are not well established, decisions regarding treatment should be based on enzymatic activity.

\section{Evaluations following initial diagnosis}

The following initial evaluations are recommended, but are not essential in all cases, for individuals identified as probably having and those confirmed as having biotinidase deficiency:

- Confirmatory quantitative determination of biotinidase enzyme activity in serum/plasma.

- Mutation analysis (genotyping) pending ambiguity of diagnosis by enzymatic activity.

- Identification of biochemical abnormalities such as metabolic ketolactic acidosis, hyperammonemia, and organic aciduria. This is not essential in infants identified by newborn screening; however, it should be considered in a symptomatic child who is not being treated with biotin or if there are concerns about noncompliance.

- Determination of plasma and/or urinary free and esterified carnitine may also be useful in symptomatic children, especially if the individual has organic acidemia/uria.

- Identification of cellular immunologic abnormalities. Once again, this should be considered when there are concerns about noncompliance.

- Physical examination for hypotonia; ataxia; eye findings, such as optic atrophy; eczematous skin rash; alopecia; conjunctivitis; breathing abnormalities, such as stridor, thrush, and/or candidiasis. This should be considered when there are concerns about noncompliance.

- Audiological evaluation for sensorineural hearing loss.

- Ophthalmological evaluation for optic atrophy and other eye abnormalities.

- Evaluation for developmental and/or psychomotor deficits.

\section{Prevention of secondary complications: surveillance}

The following evaluations are recommended, but not essential for all cases, for the surveillance of individuals with biotinidase deficiency:

- Regularly scheduled appointments with primary-care physicians or as needed.

- Annual ophthalmologic examinations (biannual for partial deficiency). Because we do not have clear long-term out- come information, periodic evaluations are recommended. Less frequent evaluations are recommended for individuals with partial deficiency.

- Annual auditory evaluations (every 2 years for partial deficiency). Again, because we do not have clear long-term outcome information, periodic evaluations are recommended. Less frequent evaluations are recommended for individuals with partial deficiency.

- Annual evaluation by a medical geneticist or metabolic specialist. Less frequent evaluations are recommended for individuals with partial deficiency.

- Symptomatic children with residual clinical problems should be seen by the appropriate subspecialists.

- If symptoms develop or return while the patient is apparently on biotin therapy, urinary organic acids should be evaluated; this problem is most commonly due to noncompliance with biotin therapy.

- As discussed earlier, determination of biotin concentrations in plasma or urine is not useful except to determine compliance with therapy. When children are taking biotin, their biotin concentrations are usually markedly elevated. If their concentrations are normal or low, it is probably due to noncompliance.

\section{DIFFERENTIAL DIAGNOSIS}

Clinical features such as vomiting, hypotonia, and seizures accompanied by metabolic ketolactic acidosis or mild hyperammonemia are often observed in inherited metabolic diseases. ${ }^{5}$ Individuals with biotinidase deficiency may exhibit clinical features that are misdiagnosed as other disorders, such as isolated carboxylase deficiency, before they are correctly identified. ${ }^{50,51}$ Other symptoms that are more characteristic of biotinidase deficiency (e.g., skin rash, alopecia) can also occur in children with nutritional biotin deficiency, holocarboxylase synthetase deficiency, zinc deficiency, or essential fatty acid deficiency.

Sensorineural hearing loss has many causes. Biotinidase deficiency can be excluded as a cause of deafness by determining biotinidase enzyme activity in serum. Testing for biotinidase deficiency should be performed specifically on children with hearing loss who are exhibiting other clinical features consistent with biotinidase deficiency.

Ataxia also has multiple causes. Biotinidase deficiency can be excluded as a cause by determining biotinidase enzyme activity in serum. The test should especially be performed on children with ataxia who are exhibiting other clinical features consistent with biotinidase deficiency.

Biotin deficiency can usually be diagnosed by obtaining a dietary history; such individuals usually are on a diet containing large quantities of uncooked eggs (containing avidin, which binds biotin) or less likely on a diet with severely restricted quantities of foods containing biotin, especially animal products such as meats. Individuals with biotin deficiency may also have been exposed to protracted parenteral hyperalimentation without biotin supplementation. ${ }^{52-55}$ Fortunately, biotin is now routinely added to hyperalimentation solutions. Low serum 
biotin concentrations are useful in differentiating among biotin deficiency, biotinidase deficiency, and holocarboxylase synthetase deficiency, but it is important to know the method used for determining the biotin concentration. ${ }^{2}$ Only methods that distinguish biotin from biocytin or bound biotin yield reliable estimates of free biotin concentrations.

Urinary organic acid analysis is useful for differentiating isolated carboxylase deficiencies from the multiple carboxylase deficiencies that occur in biotinidase deficiency and holocarboxylase synthetase deficiency. $\beta$-Hydroxyisovalerate is the most commonly elevated urinary metabolite in biotinidase deficiency, holocarboxylase synthetase deficiency, isolated $\beta$-methylcrotonyl-CoA carboxylase deficiency, and acquired biotin deficiency. In addition to $\beta$-hydroxyisovalerate, elevated concentrations of urinary lactate, methylcitrate, and $\beta$-hydroxypropionate are indicative of multiple carboxylase deficiency.

The multiple carboxylase deficiencies, biotinidase deficiency and holocarboxylase deficiency, are biotin-responsive, whereas the isolated carboxylase deficiencies are not. A trial of biotin can be useful for discriminating between the disorders. Isolated carboxylase deficiency can be diagnosed by demonstrating deficient enzyme activity of one of the three mitochondrial carboxylases in peripheral blood leukocytes (best performed prior to biotin therapy) or in cultured fibroblasts grown in low biotin-containing medium and finding normal activity of the other two carboxylases.

The symptoms of biotinidase deficiency and holocarboxylase synthetase deficiency are similar; therefore, clinical differentiation is often difficult. The age of onset of symptoms may be useful for distinguishing between holocarboxylase synthetase deficiency and biotinidase deficiency. Holocarboxylase synthetase deficiency usually presents with symptoms before the age of 3 months, whereas biotinidase deficiency usually initially presents after the age of 3 months; however, there are exceptions for both disorders.

Organic acid abnormalities in biotinidase deficiency and holocarboxylase synthetase deficiency are similar and may be reported as consistent with multiple carboxylase deficiency. In addition, tandem mass spectroscopic methodology, which is used in most newborn screening programs, can identify metabolites that are consistent with multiple carboxylase deficiency. However, although many children with holocarboxylase synthetase deficiency excrete these metabolites in the newborn period, few children with biotinidase deficiency do. Enzymatic determinations are required to distinguish definitively between the two disorders. Biotinidase determination in serum/plasma is the best and easiest way to diagnose biotinidase deficiency. Biotinidase activity is normal in serum of individuals with holocarboxylase synthetase deficiency; therefore, the enzymatic assay of biotinidase activity used in newborn screening is specific for biotinidase deficiency but does not identify children with holocarboxylase synthetase deficiency.

Prior to biotin treatment, both biotinidase deficiency and holocarboxylase synthetase deficiency are characterized by deficient activities of the three mitochondrial carboxylases in peripheral blood leukocytes. In both disorders, these activities increase to near-normal or normal after biotin treatment. However, because newborn screening for biotinidase deficiency is essentially universal in the United States, biotinidase deficiency can usually be readily excluded from the differential diagnosis.

Individuals with holocarboxylase synthetase deficiency have deficient activities of the three mitochondrial carboxylases in extracts of fibroblasts that are incubated in medium containing only the biotin contributed by fetal calf serum (low biotin), whereas individuals with biotinidase deficiency have normal carboxylase activities in fibroblasts. The activities of the carboxylases in fibroblasts of individuals with holocarboxylase synthetase deficiency usually become near-normal to normal when cultured in medium supplemented with biotin (high biotin).

\section{DETERMINATION OF BIOTINIDASE ACTIVITY IN SERUM/PLASMA}

Biotinidase activity in serum is commonly determined colorimetrically by measuring the release of $p$-aminobenzoate from $N$-biotinyl- $p$-aminobenzoate, an analog of biocytin. ${ }^{6}$ Biotinidase activity can also be determined fluorimetrically by measuring the release of aminoquinoline from biotinyl-6-aminoquinoline. ${ }^{56}$ Other assays for biotinidase activity in serum and tissues measure the hydrolysis of biocytin or other biotinyl derivatives. ${ }^{57,58}$

Individuals with profound biotinidase deficiency have lower than $10 \%$ mean normal serum enzyme activity. Individuals with partial biotinidase deficiency have $10-30 \%$ of mean normal serum biotinidase activity. Individuals with either profound biotinidase deficiency or partial biotinidase deficiency are usually identified by newborn screening in states in which it is offered. ${ }^{42,43}$ Deficient biotinidase activity has also been shown in extracts of leukocytes and fibroblasts of individuals with biotinidase deficiency. ${ }^{30}$

\section{NEWBORN SCREENING}

Biotinidase deficiency can be detected in essentially all affected infants by newborn screening. Newborn screening for biotinidase deficiency is performed in almost all states in the United States and in many countries. The working group of the American College of Medical Genetics Laboratory Quality Assurance Committee has established technical standards and guidelines for the diagnosis of biotinidase deficiency by newborn screening. ${ }^{59}$

\section{Methodologies and limitations}

Newborn screening for biotinidase deficiency requires measurement of biotinidase activity in blood-saturated filter paper samples used for all other newborn screening tests. Enzyme activity is either determined using a colorimetric assay, in which biotiniyl-p-aminobenzoate is the substrate, or using a fluorimetric assay, in which biotinyl-6-aminoquinoline is usually the substrate. ${ }^{15,60-63}$ If an individual is being treated with sulfa medications, false-negative results can occur when using the assay where biotinyl-p-aminobenzoate is the substrate $^{15}$; fortunately, sulfa medications are contraindicated for 
pregnant women and infants. False-positive results can occur when using the assay where biotinyl-6-aminoquinoline is the substrate, if an individual has an above-normal albumin concentration or is being treated with valproic acid or certain antibiotics; false-negative results can occur if an individual has an elevated triglyceride concentration, due to the administration of intravenous lipids, or immunoglobulin concentration. ${ }^{64}$ These tests are semiquantitative or quantitative. Some of these methodologies have been adapted to commercially available assay kits. However, these neonatal screening tests should not be used to differentiate partial from profound biotinidase deficiency. If enzymatic activity is low, confirmatory quantitative biotinidase activity should be determined in serum or plasma.

False-positive screening test results occur more commonly in samples from premature infants and when blood-saturated screening cards are placed in plastic envelopes or mailers before they are sufficiently dry.

I am aware of several children with profound biotinidase deficiency who have been missed on newborn screening (falsenegatives) and ultimately developed symptoms of disorder. Therefore, if a child develops symptoms consistent with the disorder, especially organic aciduria, even though the newborn screening test result was reported as normal, it is warranted to repeat enzymatic testing for biotinidase deficiency to confirm that the child does not, in fact, have the disorder. If the biotinidase activity is confirmed to be normal, holocarboxylase synthetase deficiency should be considered.

\section{Incidence}

Based on the results of worldwide screening of biotinidase deficiency, ${ }^{62}$ the incidence of the disorder is about 1 in 137,000 for profound biotinidase deficiency and 1 in about 110,000 for partial biotinidase deficiency, or 1 in about 61,000 for the combined incidence of profound and partial biotinidase deficiency. There has not been a more recent assessment of incidence either in the United States or worldwide. Based on the incidence of biotinidase deficiency determined by newborn screening, the carrier frequency in the general population is estimated at about 1 in 120 .

\section{MOLECULAR GENETICS OF BIOTINIDASE DEFICIENCY}

More than 150 mutations have been reported in children with profound biotinidase deficiency. ${ }^{65-68}$ These mutations include missense, nonsense, deletion, insertions, double-allelic, and splice-junction mutations. Mutations have been found throughout the gene, with some clustering at the carboxy-terminus. ${ }^{68}$ Many of the missense mutations occur within conserved regions of the enzyme ${ }^{69}$ and/or the tertiary structure of the enzyme. ${ }^{17}$ Six different mutation combinations account for about $60 \%$ of the mutations causing profound and partial biotinidase deficiency (Table 1). ${ }^{70}$ These include the following:

A seven-base deletion/three-base insertion mutation, c.98_104delinsTCC (G98del3ins), causes a frameshift
Table 1 Most common pathologic allelic variants of BTD

\begin{tabular}{ll}
$\begin{array}{l}\text { Nucleotide change(s) } \\
\text { in the gene }\end{array}$ & $\begin{array}{l}\text { Amino acid change } \\
\text { in the enzyme }\end{array}$ \\
\hline c. 98 104delinsTCC (G98del3ins) & p.Cys33PhefsX36 \\
\hline c. $511 G>A ;$; $1330 G>C$ & p.Ala171Thr; p.Asp444His \\
\hline c. $1368 A>C$ & p.Gln456His \\
\hline c. $1612 C>T$ & p.Arg538Cys \\
c. $1330 G>C$ & p.Asp444His \\
\hline
\end{tabular}

(p.Cys33PhefsX36) that occurs in at least one allele of the biotinidase gene in $\sim 50 \%$ of symptomatic children in the United States. ${ }^{71}$ A missense mutation, c.1612C $>$ T, p.Arg538Cys, which is located at the carboxy-terminus of the enzyme, is the second most common cause of profound biotinidase deficiency in symptomatic children in the United States. ${ }^{72}$ A c.1368A $>\mathrm{C}$ substitution results in a missense mutation, p.Gln456 His. ${ }^{73}$ c.511G $>A$ in cis combination with c.1330G $>C$ causes p.(Ala171Thr;Asp444His), resulting in a profound biotinidase deficiency allele. ${ }^{74}$ An allele with the double mutation combined with a second allele for profound biotinidase deficiency causes profound biotinidase deficiency. The latter two mutations have been reported in many children identified by newborn screening who had profound biotinidase deficiency. ${ }^{70}$

Individuals who are compound heterozygous for only the p.Asp444His mutation and a mutation that causes profound biotinidase deficiency are expected to have $\sim 20-25 \%$ of mean normal serum biotinidase activity, or partial biotinidase deficiency. ${ }^{75}$ More than $98 \%$ of the individuals with partial biotinidase deficiency have the p.Asp444His mutation as one of their alleles.

Individuals who are homozygous for the p.Asp444His mutation are expected to have $\sim 50 \%$ of mean normal serum biotinidase deficiency. This degree of activity is similar to that of individuals heterozygous for profound biotinidase deficiency. Because untreated heterozygotes for profound biotinidase deficiency do not develop symptoms, no treatment is recommended for individuals who are homozygous for the p.Asp444His mutation.

Normal allelic variants or polymorphisms have been found among individuals with normal biotinidase activity.

\section{Molecular testing for biotinidase deficiency}

There are several options for genotyping individuals for biotinidase deficiency (see Table 2). These include targeted mutation analysis, complete gene sequencing, and, potentially, deletion/ duplication analysis.

Targeted mutation analysis. Targeted mutation analysis is commercially available. One laboratory, ARUP, uses polymerase chain reactions followed by single-nucleotide extension and capillary electrophoresis of whole blood to determine the four common mutations causing profound biotinidase deficiency, including the one double allelic mutation (p.Cys33PhefsX36, p.Gln456His, p.Arg538Cys, and 
Table 2 Summary of molecular testing options for biotinidase deficiency

\begin{tabular}{|c|c|c|c|}
\hline $\begin{array}{c}\text { Gene } \\
\text { symbol }\end{array}$ & $\begin{array}{c}\text { Test } \\
\text { method }\end{array}$ & Mutations detected & $\begin{array}{l}\text { Mutation } \\
\text { detection } \\
\text { frequency by } \\
\text { test method }\end{array}$ \\
\hline \multirow[t]{3}{*}{$B T D$} & $\begin{array}{l}\text { Targeted } \\
\text { mutation } \\
\text { analysis }\end{array}$ & $\begin{array}{l}\text { p.Cys33PhefsX36, } \\
\text { p.Gln456His, p.Arg538Cys, } \\
\text { p.Asp444His, } \\
\text { p.(Ala171Thr;Asp444His), and } \\
\text { p.(Phe403vVal;Asp444His)b, } \\
\text { p.(Arg157His;Asp444His) }\end{array}$ & $\sim 60 \%$ \\
\hline & $\begin{array}{l}\text { Sequence } \\
\text { analysis }\end{array}$ & Sequence variantsc & $\sim 99 \%$ \\
\hline & $\begin{array}{l}\text { Deletion/ } \\
\text { duplication } \\
\text { analysisc }\end{array}$ & $\begin{array}{l}\text { Partial and whole-gene } \\
\text { deletions }\end{array}$ & Unknown ${ }^{d}$ \\
\hline
\end{tabular}

aExamples of mutations detected by sequence analysis may include missense, nonsense, splice-site mutations, and small intragenic deletions/insertions.

${ }^{b}$ Additional mutations available at PerkinElmer Genetics. 'Testing that detects deletions/duplications not readily detectable by sequence analysis of genomic DNA; a variety of methods including quantitative $P C R$, real-time PCR, multiplex ligation-dependent probe amplification, and comparative genomic hybridization microarray analysis can be used. ${ }^{d} \mathrm{No}$ large deletions have been reported in BTD. Based on the high sensitivity of BTD sequence analysis, a screening test for large deletions may not be warranted.

p.(Ala171Thr;Asp444His)), and the common allele for partial biotinidase deficiency (p.Asp444His). The four common mutations causing profound biotinidase deficiency and the common allele for partial biotinidase deficiency comprise $\sim 60 \%$ of the abnormal alleles found in symptomatic individuals and in children identified by newborn screening in the United States. ${ }^{65,70}$ Another laboratory, PerkinElmer Genetics, uses real-time polymerase chain reaction of DNA isolated from the blood spot of a newborn screen card to identify the same four common mutations causing profound biotinidase deficiency and the single mutation causing partial biotinidase deficiency. In addition to the one double mutation causing profound biotinidase deficiency cited above (p.(Ala171Thr;Asp444His)), this laboratory also tests for two other mutations that in combination with the Asp444His mutation, p.(Phe403vVal;Asp444His) and p.(Arg157His;Asp444His), cause profound biotinidase deficiency. ${ }^{68}$ The latter targeted mutation panel allows more definitive differentiation of individuals having the Asp444His mutation causing partial biotinidase deficiency from those having profound biotinidase deficiency.

Using the results of either panel in combination with enzymatic data should be sufficient to determine an individual's status. Targeted mutation analysis is less expensive than complete sequencing of the gene but fails to identify less common mutations causing biotinidase deficiency. However, it is useful in determining if an individual has partial biotinidase deficiency. Essentially all individuals with partial deficiency have the p.Asp444His mutation. ${ }^{75}$ If a child has this mutation, does not have the Ala171Thr mutation or one of two other rare mutations that in combination with the p.Asp444His mutation cause profound deficiency, and has more enzymatic activity than seen in those with profound deficiency, this analysis should confirm the diagnosis of partial biotinidase deficiency in the child, especially with ambiguous enzyme results.

Gene sequence analysis. Direct sequencing of BTD and its intron/exon junctions is available clinically. ${ }^{46,76}$ Almost all individuals with partial biotinidase deficiency have the mutation p.Asp444His in one allele of BTD in combination with a mutation for profound deficiency in the other allele. ${ }^{75}$

In our experience, complete sequencing will ascertain both mutations causing profound and those causing partial biotinidase deficiency in essentially all individuals with corresponding enzymatic activity indicative of profound or partial deficiency. In addition, complete gene sequence analysis will help to confirm or exclude status in an individual with ambiguous enzymatic results.

Deletion/duplication analysis. Although deletion testing is offered clinically, as of this writing, no large deletions have been reported in BTD. Based on the high sensitivity of BTD sequence analysis, a screening test for large deletions/duplications may not be warranted.

Interpretation of molecular test results. There is a recent compilation of mutations causing biotinidase deficiency. ${ }^{68}$ Mutations are continually updated in a database on the following Internet site: (http://www.arup.utah.edu/database/BTD/BTD_ welcome.php)..$^{77}$

\section{TESTING STRATEGIES}

\section{Confirmation of the diagnosis of a proband}

A child with clinical features suggestive of biotinidase deficiency or whose biochemical findings are indicative of multiple carboxylase deficiency should have quantitative biotinidase enzyme activity determined in serum/plasma. With appropriate controls and handling of samples, this testing is usually definitive for confirming the diagnosis.

A child who is identified by newborn screening as putatively having biotinidase deficiency should have quantitative determination of biotinidase enzyme activity in serum/plasma to confirm the diagnosis.

Molecular genetic testing of BTD is most useful when the results of enzymatic testing are ambiguous. For example, such analysis can differentiate a child with profound biotinidase deficiency from one with partial biotinidase deficiency or a child with partial biotinidase deficiency from one who is heterozygous for profound biotinidase deficiency or homozygous for the p.Asp444His mutation.

\section{Carrier testing for at-risk individuals}

Heterozygotes usually have serum enzyme activities intermediate between those of affected and those of normal individuals. ${ }^{6,78}$ Individuals who are heterozygous for biotinidase deficiency are not at risk of developing symptoms of the disorder. Enzymatic 
activity in serum/plasma can identify heterozygotes with about 95\% accuracy, especially when performed simultaneously with samples from known carriers, such as parents of an individual with profound biotinidase deficiency ${ }^{79}$ However, mutation analysis can readily establish the carrier status of an individual, especially if the disease-causing mutations are known in an affected proband.

\section{GENOTYPE-PHENOTYPE CORRELATIONS}

Genotype-phenotype correlations are not well established in biotinidase deficiency. Deletions, insertions, or nonsense mutations usually result in complete absence of biotinidase enzyme activity, whereas missense mutations may or may not result in complete loss of enzymatic activity. Those with total absence of biotinidase activity may be more likely to develop symptoms than an individual who has some residual enzyme activity; however, all individuals with profound biotinidase deficiency require biotin treatment.

Most mutations in BTD cause complete loss or nearly complete loss of biotinidase enzyme activity. The affected alleles are considered profound biotinidase deficiency alleles; a combination of two such alleles, whether homozygous or compound heterozygous, results in the individual having profound biotinidase deficiency. Such individuals are likely to develop symptoms if not treated with biotin.

Individuals with one profound biotinidase deficiency allele and a normal allele are heterozygotes or carriers of profound biotinidase deficiency. Parents of children with profound biotinidase deficiency are in this group. No heterozygous parents of children with profound or partial biotinidase deficiency have exhibited symptoms (unpublished data). Such individuals do not need biotin therapy.

One allele with both the p.Asp444His and p.Ala171Thr mutations in cis configuration p.(Ala171Thr;Asp444His) (compound mutation) results in profound biotinidase deficiency. An individual with an allele having this compound mutation combined with another allele with a mutation for profound biotinidase deficiency has profound biotinidase deficiency and requires biotin therapy. ${ }^{74}$

Individuals who are compound heterozygotes for the p.Asp444His mutation and a mutation that results in profound biotinidase deficiency are expected to have $\sim 20-25 \%$ of mean normal serum biotinidase enzyme activity, or partial biotinidase deficiency. ${ }^{75}$ Individuals in this group are routinely treated with biotin. ${ }^{42}$ Individuals who are homozygous or have two alleles for the p.Asp444His mutation are expected to have $~ 50 \%$ of mean normal serum biotinidase enzyme activity. This enzyme activity is similar to that of individuals who are heterozygous for profound biotinidase deficiency. Such individuals do not need biotin therapy.

Several adults with profound biotinidase deficiency have never had symptoms and have never been treated, ${ }^{44}$ whereas some children with the same mutations have been symptomatic. Therefore, it has been speculated that some children with profound biotinidase deficiency may exhibit mild or no symptoms if left untreated. However, it is recommended that these children be treated. ${ }^{48}$

Although genotype-phenotype correlations are not well established, in one study, children with symptoms of profound biotinidase deficiency with null mutations were more likely to develop hearing loss than those with missense mutations, even if not treated for a period of time. ${ }^{80}$ Because newborn screening for biotinidase deficiency is almost universal and children with biotinidase deficiency are treated from birth, it is likely that we will not be able to ascertain more about the natural history of the disorder in symptomatic individuals in the future. Therefore, further genotype-phenotype correlations are unlikely to be forthcoming.

\section{PRENATAL TESTING AND COUNSELING}

Because biotinidase deficiency is readily treatable, requests for prenatal diagnosis are not common. Differences in perspective may exist among medical professionals and in families regarding the use of prenatal testing, particularly if the testing is being considered for the purpose of pregnancy termination rather than early diagnosis. Although most centers would consider decisions about prenatal testing to be the choice of the parents, discussion of these issues is appropriate. In my experience, it is the parents of severely symptomatic children with the disorder who are more likely to request or consider prenatal diagnosis than those who have enzyme-deficient children identified by newborn screening and were treated before they developed symptoms.

\section{Enzymatic testing}

Prenatal diagnosis for pregnancies at increased risk is possible by measuring biotinidase enzyme activity in cultured amniotic fluid cells and in amniotic fluid obtained by amniocentesis at $\sim 15-18$ weeks of gestation. ${ }^{81,82}$

\section{Molecular genetic testing}

Prenatal diagnosis for pregnancies at increased risk is also possible by analysis of DNA extracted from fetal cells obtained by amniocentesis at $\sim 15-18$ weeks of gestation or by chorionic villus sampling at $\sim 10-12$ weeks of gestation. ${ }^{83}$ Both diseasecausing alleles of an affected family member must be identified before molecular analysis can be performed.

\section{Preimplantation genetic diagnosis}

Preimplantation genetic diagnosis may be available for families in which the disease-causing mutations have been identified. However, because immediate or early biotin treatment of a newborn child with biotinidase deficiency apparently prevents symptoms, requests for preimplantation genetic diagnosis for biotinidase deficiency will probably not be common.

\section{Pregnancy management}

There are no special pregnancy management issues for a woman who is carrying a baby with biotinidase deficiency or who is at risk of having a baby with biotinidase deficiency. However, consideration should be given to supplementing the mother with some biotin during the pregnancy. 


\section{EMERGING ISSUES}

Ambiguity in interpreting enzymatic biotinidase activity results

Although most children with profound biotinidase deficiency are diagnosed correctly by enzymatic determination in serum or plasma, I am aware of several individuals who were inappropriately diagnosed with profound biotinidase deficiency. These children were subsequently found to have normal enzyme activity after complete gene sequencing failed to identify a mutation. The problem in unambiguously determining biotinidase deficiency is almost certainly due to enzyme deterioration resulting from inappropriate storage of samples at the site of the blood draw, at the laboratory storing the sample prior to shipping, or at the laboratory performing the testing. ${ }^{46}$ In addition, many laboratories, especially commercial laboratories, request and receive a sample from only the proband. Therefore, if an individual has low enzymatic activity, it is difficult, if not impossible, to interpret the result because there are no internal controls with which to compare this activity, only the laboratory's external control. It has been repeatedly recommended that sera be immediately separated from the whole blood, frozen, shipped on dry ice, and stored at $-80^{\circ} \mathrm{C}$ at the assaying laboratory until tested. ${ }^{8}$ In addition, it has been recommended that confirmatory testing should include samples from the proband, parents, and an unrelated control. If this protocol is followed, the results of the enzyme determinations can be interpreted more accurately. ${ }^{84}$

Difficulty in interpreting enzymatic activity results also can occur when differentiating individuals with partial deficiency from those who are heterozygous for profound biotinidase deficiency, and those who are heterozygous for profound biotinidase deficiency from individuals with normal activity. Individuals who are heterozygous for partial biotinidase deficiency usually have about $75 \%$ of mean normal activity, which overlaps considerably with the normal range of activity. Carriers for profound biotinidase deficiency can more easily be determined when their activities are compared with those of the various family members and the unrelated control, all obtained, shipped, and assayed at the same time. Further clarification of status of a child suspected of having the disorder or as being a carrier may require genotyping.

The recommendations of sending samples immediately from the family and a control collected and stored under optimal frozen conditions will permit more accurate interpretation of results. These recommendations are often not heeded because it is generally considered impractical. However, continuing the current practices will likely propagate ambiguity in enzyme interpretation.

\section{False-positive rate in newborn screening for biotinidase deficiency}

During the 1980s and the 1990s, the colorimetric method was almost always used for newborn screening of biotinidase deficiency. This semiquantitative method was simply intended to identify children with putative biotinidase deficiency. The primary purpose of all newborn screening tests are to only identify which infants likely are affected and confirmatory studies are then used to confirm the diagnosis. At that time, biotinidase deficiency screening had one of the lowest falsepositive rates as compared with other disorders screened. ${ }^{15,85}$ However, over the past 5 to 10 years, it appears that the falsepositive rate for biotinidase deficiency screening has increased markedly in many newborn screening programs. Various factors may explain this increased rate, but the likely cause is that there have been numerous modifications made to the methods by various newborn screening laboratories and by the companies supplying the commercial kits. These alterations, for the most part, were to make the assay more quantitative by applying various "cutoff" values to identify and differentiate affected children. Although the manufacturers of these kits usually recommended that each laboratory establish its own cutoff values, many laboratories failed to do this and accepted the kit's cutoff values. The quantitative fluorescent substrate methods and kits will probably result in similar problems. Until the cause of the increase in false-positive rate is clarified and corrected, it is likely to remain relatively high compared with many other disorders screened.

It must be emphasized that newborn screening for a disorder is not meant to be the method to determine definitive enzyme status, but rather to identify children who putatively have the disorder. Once such a child is identified as having possible biotinidase deficiency, carefully conducted quantitative enzymatic testing should be performed to confirm the diagnosis. This will undoubtedly result in fewer families experiencing the anxiety that accompanies an increased falsepositive rate and fewer health professionals experiencing the frustration of interpreting ambiguous results. However, because of the aforementioned complications, the definitive status of individuals suspected of having biotinidase deficiency or those who are carriers for the disorder may require mutation analysis.

\section{CONCLUSION}

Symptomatic individuals with biotinidase deficiency can be readily and easily treated with biotin. Symptoms can be prevented if affected individuals are treated early. Because newborn screening for biotinidase deficiency meets the major criteria for inclusion of screening of the disorder in most newborn screening programs, it is being universally adopted in the United States and around the world. Although newborn screening for biotinidase deficiency can identify affected children, allow for early treatment, and prevent the development of symptoms in these children, newborn screening will obviously curtail our further understanding of the natural history of the disorder. In addition, it is unlikely that we will be able to obtain more information about genotype-phenotype relationships. Nevertheless, biotinidase deficiency is an excellent example of the early identification and successful treatment of a genetic disease. Therefore, "if you have to have an inherited metabolic disease, biotinidase deficiency is the one to have!" 


\section{ACKNOWLEDGMENT}

I thank the Safra Research Fund for their support of this review and of my research.

\section{DISCLOSURE}

The author declares no conflict of interest.

\section{REFERENCES}

1. Pispa J. Animal biotinidase. Ann Med Exp Biol Fenn 1965;43(suppl 5):1-39.

2. Wolf B. Disorders of biotin metabolism. In: Scriver CR, Beaudet AL, Sly WS, Valle D (eds). The Metabolic and Molecular Bases of Inherited Disease. McGraw-Hill: New York, 2001:3935-3962.

3. Craft DV, Goss NH, Chandramouli N, Wood HG. Purification of biotinidase from human plasma and its activity on biotinyl peptides. Biochemistry 1985:24:2471-2476.

4. Bonjour JP. Biotin in man's nutrition and therapy - a review. Int J Vitam Nutr Res 1977:47:107-118.

5. Wolf B, Hsia YE, Sweetman L, et al. Multiple carboxylase deficiency: clinical and biochemical improvement following neonatal biotin treatment. Pediatrics 1981;68:113-118.

6. Wolf B, Grier RE, Allen RJ, Goodman SI, Kien CL. Biotinidase deficiency: the enzymatic defect in late-onset multiple carboxylase deficiency. Clin Chim Acta 1983;131:273-281

7. Wolf B, Grier RE, Secor McVoy JR, Heard GS. Biotinidase deficiency: a novel vitamin recycling defect. J Inherit Metab Dis 1985;8(suppl 1):53-58.

8. Wolf $B$. Clinical issues and frequent questions about biotinidase deficiency. Mol Genet Metab 2010;100:6-13.

9. Hymes J, Wolf B. Biotinidase and its roles in biotin metabolism. Clin Chim Acta 1996;255:1-11.

10. Wolf B, Miller JB, Hymes J, Secor McVoy J, Ishikawa Y, Shapira E. Immunological comparison of biotinidase in serum from normal and biotinidase-deficient individuals. Clin Chim Acta 1987;164:27-32.

11. Chauhan J, Dakshinamurti K. Purification and characterization of human serum biotinidase. J Biol Chem 1986;261:4268-4275.

12. Hart PS, Hymes J, Wolf B. Isoforms of human serum biotinidase. Clin Chim Acta 1991;197:257-264.

13. Oizumi J, Hayakawa K, Hosoya M. Comparative study on human milk and serum biotinidase. Biochimie 1989;71:1163-1169.

14. Knappe J, Bruemmer W, Biederbick K. [Purification and properties of biotinidase from swine kidney and lactobacillus casei]. Biochem Z 1963:338:599-613.

15. Heard GS, Secor McVoy JR, Wolf B. A screening method for biotinidase deficiency in newborns. Clin Chem 1984;30:125-127.

16. Hymes J, Fleischhauer K, Wolf B. Biotinylation of histones by human serum biotinidase: assessment of biotinyl-transferase activity in sera from normal individuals and children with biotinidase deficiency. Biochem Mol Med 1995:56:76-83.

17. Pindolia K, Jensen $K$, Wolf $B$. Three dimensional structure of human biotinidase: computer modeling and functional correlations. Mol Genet Metab 2007;92:13-22.

18. Hart PS, Hymes J, Wolf B. Biochemical and immunologic characterization of serum biotinidase in partial biotinidase deficiency. Pediatr Res 1992;31:261-265

19. Hart PS, Barnstein BO, Secor McVoy JR, Matalon R, Wolf B. Comparison of profound biotinidase deficiency in children ascertained clinically and by newborn screening using a simple method of accurately determining residual biotinidase activity. Biochem Med Metab Biol 1992;48:41-45.

20. Cole H, Weremowicz S, Morton CC, Wolf B. Localization of serum biotinidase (BTD) to human chromosome 3 in band p25. Genomics 1994;22:662-663.

21. Cole H. Cloning and characterization of the human biotinidase gene. Thesis, Medical College of Virginia/Virginia Commonwealth University: Richmond, VA, 1994.

22. Knight HC, Reynolds TR, Meyers GA, Pomponio RJ, Buck GA, Wolf $B$. Structure of the human biotinidase gene. Mamm Genome 1998;9: 327-330.

23. Cole H, Reynolds TR, Lockyer JM, et al. Human serum biotinidase. cDNA cloning, sequence, and characterization. J Biol Chem 1994;269: 6566-6570.

24. Wolf B, Grier RE, Allen RJ, et al. Phenotypic variation in biotinidase deficiency. J Pediatr 1983;103:233-237.
25. Wolf B, Heard GS, Weissbecker KA, McVoy JR, Grier RE, Leshner RT Biotinidase deficiency: initial clinical features and rapid diagnosis. Ann Neurol 1985;18:614-617.

26. Wastell HJ, Bartlett K, Dale G, Shein A. Biotinidase deficiency: a survey of 10 cases. Arch Dis Child 1988;63:1244-1249.

27. Wolf B. The neurology of biotinidase deficiency. Mol Genet Metab 2011;104:27-34.

28. Salbert BA, Pellock JM, Wolf B. Characterization of seizures associated with biotinidase deficiency. Neurology 1993;43:1351-1355.

29. Chedrawi AK, Ali A, Al Hassnan ZN, Faiyaz-Ul-Haque M, Wolf B. Profound biotinidase deficiency in a child with predominantly spinal cord disease. J Child Neurol 2008;23:1043-1048.

30. Wolf $B$, Secor McVoy J. A sensitive radioassay for biotinidase activity: deficient activity in tissues of serum biotinidase-deficient individuals. Clin Chim Acta 1983;135:275-281.

31. Lott IT, Lottenberg S, Nyhan WL, Buchsbaum MJ. Cerebral metabolic change after treatment in biotinidase deficiency. J Inherit Metab Dis 1993;16: 399-407.

32. Grünewald S, Champion MP, Leonard JV, Schaper J, Morris AA. Biotinidase deficiency: a treatable leukoencephalopathy. Neuropediatrics 2004;35: 211-216

33. Taitz LS, Leonard JV, Bartlett K. Long-term auditory and visual complications of biotinidase deficiency. Early Hum Dev 1985;11:325-331.

34. Salbert BA, Astruc J, Wolf B. Ophthalmologic findings in biotinidase deficiency. Ophthalmologica 1993;206:177-181.

35. Weber P, Scholl S, Baumgartner ER. Outcome in patients with profound biotinidase deficiency: relevance of newborn screening. Dev Med Child Neurol 2004;46:481-484.

36. Wolf $B$, Spencer R, Gleason T. Hearing loss is a common feature of symptomatic children with profound biotinidase deficiency. J Pediatr 2002;140:242-246.

37. Burton BK, Roach ES, Wolf B, Weissbecker KA. Sudden death associated with biotinidase deficiency. Pediatrics 1987;79:482-483.

38. Ramaekers VT, Brab M, Rau G, Heimann G. Recovery from neurological deficits following biotin treatment in a biotinidase $\mathrm{Km}$ variant. Neuropediatrics 1993;24:98-102.

39. Suormala T, Ramaekers VT, Schweitzer S, et al. Biotinidase Km-variants: detection and detailed biochemical investigations. J Inherit Metab Dis 1995;18:689-700.

40. Tokatli A, Coskun T, Ozalp I. Biotinidase deficiency with neurological features resembling multiple sclerosis. J Inherit Metab Dis 1997;20:707-708.

41. Wolf B, Pomponio RJ, Norrgard KJ, et al. Delayed-onset profound biotinidase deficiency. J Pediatr 1998;132:362-365.

42. McVoy JR, Levy HL, Lawler M, et al. Partial biotinidase deficiency: clinical and biochemical features. J Pediatr 1990;116:78-83.

43. Suormala TM, Baumgartner ER, Wick H, Scheibenreiter S, Schweitzer S. Comparison of patients with complete and partial biotinidase deficiency: biochemical studies. J Inherit Metab Dis 1990;13:76-92.

44. Wolf B, Norrgard K, Pomponio RJ, et al. Profound biotinidase deficiency in two asymptomatic adults. Am J Med Genet 1997;73:5-9.

45. Baykal T, Gokcay G, Gokdemir Y, et al. Asymptomatic adults and older siblings with biotinidase deficiency ascertained by family studies of index cases. J Inherit Metab Dis 2005;28:903-912.

46. Wolf B. Biotinidase deficiency: new directions and practical concerns. Curr Treat Options Neurol 2003;5:321-328.

47. Moslinger D, Stockler-Ipsiroglu S, Scheibenreiter S, et al. Clinical and neuropsychological outcome in 33 patients with biotinidase deficiency ascertained by nationwide newborn screening and family studies in Austria. Eur J Pediatr 2002;161:167-168.

48. Möslinger D, Mühl A, Suormala T, Baumgartner R, Stöckler-lpsiroglu S. Molecular characterisation and neuropsychological outcome of 21 patients with profound biotinidase deficiency detected by newborn screening and family studies. Eur J Pediatr 2003;162(suppl 1):S46-S49.

49. Wolf $B$. Children with profound biotinidase deficiency should be treated with biotin regardless of their residual enzyme activity or genotype. Eur J Pediatr 2002;161:167-8; author reply 169.

50. Suormala T, Wick H, Bonjour JP, Baumgartner ER. Rapid differential diagnosis of carboxylase deficiencies and evaluation for biotin-responsiveness in a single blood sample. Clin Chim Acta 1985;145:151-162.

51. Wolf B. Disorders of biotin metabolism. In: Scriver CR, Beaudet AL, Sly WS, Valle D (eds). The Metabolic Basis of Inherited Disease. McGraw-Hill: New York, 1992:2083-2103. 
52. Mock DM, Baswell DL, Baker H, Holman RT, Sweetman L. Biotin deficiency complicating parenteral alimentation: diagnosis, metabolic repercussions, and treatment. Ann NY Acad Sci 1985;447:314-334.

53. Swick HM, Kien CL. Biotin deficiency with neurologic and cutaneous manifestations but without organic aciduria. J Pediatr 1983;103:265-267.

54. Innes SM, Allardya DB. Possible biotin deficiency in adults receiving longterm total parenteral nutrition. Am J Clin Nutr 1983;37:185.

55. McClain CJ, Baker H, Onstad GR. Biotin deficiency in an adult during home parenteral nutrition. JAMA 1982;247:3116-3117.

56. Wastell $H$, Dale $G$, Bartlett $K$. A sensitive fluorimetric rate assay for biotinidase using a new derivative of biotin, biotinyl-6-aminoquinoline. Anal Biochem 1984;140:69-73.

57. Weiner DL, Grier RE, Wolf B. A bioassay for determining biotinidase activity and for discriminating biocytin from biotin using holocarboxylase synthetase-deficient cultured fibroblasts. J Inherit Metab Dis 1985;8(suppl 2):101-102.

58. Ebrahim H, Dakshinamurti K. A fluorometric assay for biotinidase. Anal Biochem 1986;154:282-286.

59. Cowan TM, Blitzer MG, Wolf B; Working Group of the American College of Medical Genetics Laboratory Quality Assurance Committee. Technical standards and guidelines for the diagnosis of biotinidase deficiency. Genet Med 2010;12:464-470.

60. Wolf B, Heard GS, Jefferson LG, Proud VK, Nance WE, Weissbecker KA. Clinical findings in four children with biotinidase deficiency detected through a statewide neonatal screening program. N Engl J Med 1985;313:16-19.

61. Heard GS, Wolf $B$, Jefferson LG, et al. Neonatal screening for biotinidase deficiency: results of a 1-year pilot study. J Pediatr 1986;108:40-46.

62. Wolf $B$. Worldwide survey of neonatal screening for biotinidase deficiency. J Inherit Metab Dis 1991;14:923-927.

63. Pitkanen L, Tuuminen T. A quantitative fluorimetric micromethod used for the neonatal screening of biotinidase deficiency in Finland. Screening 2011;1:185-194.

64. PerkinElmer Life and Analytical Sciences. Neonatal biotinidase kit. 2011. Wallac Oy, Turku, Finland.

65. Pomponio RJ, Hymes J, Reynolds TR, et al. Mutations in the human biotinidase gene that cause profound biotinidase deficiency in symptomatic children: molecular, biochemical, and clinical analysis. Pediatr Res 1997;42:840-848.

66. Mühl A, Möslinger D, Item CB, Stöckler-lpsiroglu S. Molecular characterisation of 34 patients with biotinidase deficiency ascertained by newborn screening and family investigation. Eur J Hum Genet 2001;9:237-243.

67. Wolf $B$, Jensen $K$, Hüner $G$, et al. Seventeen novel mutations that cause profound biotinidase deficiency. Mol Genet Metab 2002;77:108-111.

68. Pindolia K, Jordan M, Wolf $\mathrm{B}$. Analysis of mutations causing biotinidase deficiency. Hum Mutat 2010;31:983-991.

69. Swango KL, Wolf B. Conservation of biotindase in mammals and identification of the putative biotinidase gene in Drosophila melanogaster. Mol Genet Metab 2001;74:492-499.
70. Norrgard KJ, Pomponio RJ, Hymes J, Wolf B. Mutations causing profound biotinidase deficiency in children ascertained by newborn screening in the United States occur at different frequencies than in symptomatic children. Pediatr Res 1999;46:20-27.

71. Pomponio RJ, Reynolds TR, Cole H, Buck GA, Wolf B. Mutational hotspot in the human biotinidase gene causes profound biotinidase deficiency. Nat Genet 1995;11:96-98.

72. Pomponio RJ, Norrgard KJ, Hymes J, et al. Arg538 to Cys mutation in a CpG dinucleotide of the human biotinidase gene is the second most common cause of profound biotinidase deficiency in symptomatic children. Hum Genet 1997;99:506-512.

73. Norrgard KJ, Pomponio RJ, Swango KL, et al. Mutation (Q456H) is the most common cause of profound biotinidase deficiency in children ascertained by newborn screening in the United States. Biochem Mol Med 1997; 61:22-27.

74. Norrgard KJ, Pomponio RJ, Swango KL, et al. Double mutation (A171T and $\mathrm{D} 444 \mathrm{H}$ ) is a common cause of profound biotinidase deficiency in children ascertained by newborn screening the the United States. Mutations in brief no. 128. Online. Hum Mutat 1998;11:410.

75. Swango KL, Demirkol M, Hüner G, et al. Partial biotinidase deficiency is usually due to the $\mathrm{D} 444 \mathrm{H}$ mutation in the biotinidase gene. Hum Genet 1998; 102:571-575.

76. Hymes J, Stanley CM, Wolf B. Mutations in BTD causing biotinidase deficiency. Hum Mutat 2001;18:375-381.

77. Procter M, Mao R, Wolf B. Online Scientific ResourceARUP: Biotinidase deficiency and BTD. 2011. http://www.arup.utah.edu/database/BTD/BTD_ welcome.php.

78. Weissbecker KA, Nance WE, Eaves LJ, Piussan C, Wolf B. Statistical approaches for the detection of heterozygotes for biotinidase deficiency. Am J Med Genet 1991;39:385-390.

79. Weissbecker KA, Nance WE, Eaves LJ, Piussan C, Wolf B. Statistical approaches for the detection of heterozygotes for biotinidase deficiency. Am J Med Genet 1991;39:385-390.

80. Sivri HS, Genç GA, Tokatli A, et al. Hearing loss in biotinidase deficiency: genotype-phenotype correlation. J Pediatr 2007;150:439-442.

81. Secor McVoy JR, Heard GS, Wolf B. Potential for prenatal diagnosis of biotinidase deficiency. Prenat Diagn 1984;4:317-318.

82. Chalmers RA, Mistry J, Docherty PW, Stratton D. First trimester prenatal exclusion of biotinidase deficiency. J Inherit Metab Dis 1994;17: 751-752.

83. Pomponio RJ, Hymes J, Pandya A, et al. Prenatal diagnosis of heterozygosity for biotinidase deficiency by enzymatic and molecular analyses. Prenat Diagn 1998;18:117-122.

84. Neto EC, Schulte J, Rubim R, et al. Newborn screening for biotinidase deficiency in Brazil: biochemical and molecular characterizations. Braz J Med Biol Res 2004;37:295-299.

85. Wolf $B$, Heard GS, Jefferson LG, et al. Neonatal screening for biotinidase deficiency: an update. J Inherited Metab Dis 1986;9(suppl 2):303-306. 